\title{
Synchrotron Radiation and Beam Tube Vacuum in a Very Large Hadron Collider; Stage 1 VLHC
}

\author{
M. Pivi and W.C. Turner \\ Accelerator and Fusion Research Division \\ Ernest Orlando Lawrence Berkeley National Laboratory \\ University of California \\ Berkeley, CA-94720
}

\begin{abstract}
Synchrotron radiation induced photodesorption in particle accelerators may lead to pressure rise and to beam-gas scattering losses, finally affecting the beam lifetime. We discuss the beam tube vacuum in the low field Stage 1 Very Large Hadron Collider VLHC. Since VLHC Stage 1 has a room temperature beam tube, a non-evaporable getter (NEG St101 strip) pumping system located inside a pumping antechamber, supplemented by lumped ion pumps for pumping methane is considered. A possible beam conditioning scenario is presented for reaching design intensity. The most important results are summarized in this paper. More detailed reports of the calculations will be presented at the PAC2001 Conference, Chicago, IL to be held in June 2001, and at the Snowmass Conference, CO, to be held on July 2001.

\section{Introduction}

In the present report the required pumping speed, a possible beam current conditioning scenario, and the beam-gas scattering lifetime are discussed for Stage 1 VLHC. A self-consistent calculation is performed assuming that the beam lifetime depends on the beam tube vacuum gas pressure and on the pp collision rate at two interaction points (IPs). The vacuum tube pressure, and therefore the beam-gas scattering lifetime, is a function of the beam intensity. The parameters necessary for evaluating the beam tube vacuum in the Stage 1 VLHC are shown in Tables 1 and 2. In Table $1, \tau_{\mathrm{pp}}$ represents the proton lifetime determined by pp collisions at two IPs at the design luminosity $10^{34} \mathrm{~cm}^{-2} \mathrm{~s}^{-1}$, with the pp cross section assumed to be $\sigma_{\mathrm{pp}}=137 \mathrm{mb}$ at $40 \mathrm{TeV} \mathrm{cm}$.
\end{abstract}

Table 1. VLHC parameters for the low field Stage 1 VLHC

\begin{tabular}{llc}
\hline VLHC parameters & symbol & Stage1 \\
\hline & $E, T e V$ & 20 \\
beam energy & $B, T$ & 2 \\
dipole field at 20TeV & $C, k m$ & 232 \\
circumference & $N_{\mathrm{b}}$ & $2.48 \times 10^{10}$ \\
number of particles per bunch & $I, m A$ & 190 \\
total beam current & $L, \mathrm{~cm}^{-2} \mathrm{~s}^{-1}$ & $1 \times 10^{34}$ \\
Luminosity & $\beta, m$ & 233 \\
beta function (aver.) & $\varepsilon_{n}, \pi \mathrm{mm}-\mathrm{mrad}$ & 1.67 \\
normalized emittance & $r_{w}, \mathrm{~cm}$ & $0.9 \times 1.5$ \\
\hline & $T_{w},{ }^{o} \mathrm{~K}$ & $\sim 294$ \\
vacuum chamber dimensions & $\tau_{p p}, \mathrm{hrs}$ & 93 \\
beam tube temperature & & \\
pp collision IP lifetime & & \\
\hline
\end{tabular}


Table2: Numerical values of parameters used in the evaluation

\begin{tabular}{lccccc}
\hline gas & $\sigma_{\mathrm{pj}}$ & $\mathrm{X}_{\mathrm{oj}}$ & $\eta_{0 \mathrm{j}}$ & $v_{\mathrm{j}}$ & $\mathrm{Q}_{\mathrm{j}, \mathrm{tds}}$ \\
\hline & $(\mathrm{mb})$ & $\left(\mathrm{gm} / \mathrm{cm}^{2}\right)$ & $($ molec $/ p h)$. & & $($ nTorr-l/s-m) \\
\hline $\mathrm{H}_{2}$ & 104 & 63 & $6 \mathrm{e}-4$ & 0.8 & 6.4 \\
$\mathrm{CH}_{4}$ & 628 & 47 & $8 \mathrm{e}-5$ & 1.25 & 0.64 \\
$\mathrm{CO}$ & 960 & 38 & $2 \mathrm{e}-4$ & 0.8 & 0.128 \\
$\mathrm{CO}_{2}$ & 1500 & 36 & $1.5 \mathrm{e}-3$ & 0.8 & 0.128 \\
\hline
\end{tabular}

\section{Vacuum system for the Stage 1 VLHC}

Photodesorbed gas is the dominant gas source in the beam tube, and the photon intensity, $0.8 \times 10^{16} \mathrm{ph} / \mathrm{m}$-sec, at design current, is the most important parameter for beam tube vacuum. The synchrotron radiation power in the Stage $1 \mathrm{VLHC}, 0.033 \mathrm{~W} / \mathrm{m}$, can be absorbed by the room temperature beam tube and is low enough that active cooling is not needed.

In Stage 1 the beam-gas scattering lifetime is affected by two processes: (1) single protonnuclear collisions leading to a lost proton and (2) multiple small angle proton-nuclear Coulomb collisions leading to an increase in emittance.

The luminosity loss rate $\tau_{\mathrm{g}}$ due to beam-gas scattering is related to the proton loss rate due to collisions with gas nuclei $\tau_{\mathrm{c}}$ and the emittance growth rate due to Coulomb scattering $\tau_{\varepsilon}$ [1], and is given by:

$$
\frac{1}{\tau_{g}}=7.2 \times 10^{-6} \sum_{j} \sigma_{p j}(m b) \bar{P}_{j}(n T o r r)+\frac{1.33 \times 10^{-6} \gamma \beta(m)}{c p(T e V)^{2} \varepsilon_{n}(\pi m m-m r a d)} \sum_{j} \frac{A_{j}(g m) \bar{P}_{j}(n T o r r)}{X_{0_{j}}\left(g m / \mathrm{cm}^{2}\right)}
$$

where $A_{j}$ is the gram molecular weight, $\beta$ the average beta function $233 \mathrm{~m}$, and $\varepsilon_{n}$ the normalized emittance $1.67 \pi \mathrm{mm}$-mrad [5], the proton collision cross section $\sigma_{\mathrm{pj}}$, the radiation length $\mathrm{X}_{\mathrm{oj}}$ and the initial photodesorption coefficient $\eta_{0 \mathrm{j}}$ are listed in Table 2. For $\mathrm{H}_{2} \mathrm{O} \sigma_{\mathrm{pj}}=644 m b, \mathrm{X}_{\mathrm{oj}}=36$ $\mathrm{gm} / \mathrm{cm}^{2}$. The first term in eq. (1) is twice the proton loss rate and the second term is the emittance growth rate. Beam-gas collisions will be negligible if $\tau_{\mathrm{g}}$ is much larger than the luminosity lifetime $\tau_{\mathrm{L}}$. We will define the beam-gas scattering lifetime negligible when $\tau_{\mathrm{g}}>>5 \tau_{\mathrm{L}}$, with $\tau_{\mathrm{L}}=\tau_{\mathrm{pp}} / 2=46.5$ hrs.

Once $\tau_{\mathrm{g}}=5 \tau_{\mathrm{L}}=232 \mathrm{hrs}$ is fixed, we estimate the average beam tube gas pressure for each gas species taken separately, with the results given in Table 3. From Table 3, we can see that the CO scattering equivalent tube pressure should be less than 0.33 nTorr to reach $\tau_{\mathrm{g}}>5 \tau_{\mathrm{L}}$.

In addition to the degradation of luminosity lifetime, we have to consider the consequences of the beam-gas scattered beam power which can lead to undesirable heat loads and activation of storage ring components. We estimate the beam tube pressure that would result in a scattered beam power of $0.1 \mathrm{~W} / \mathrm{m}$, corresponding to a typical limit for the global capacity of a cryogenic refrigeration plant. Results for each gas species are given in Table 3, where we can see that the pressure limiting factor is given by beam-gas scattering particle loss rather than the power loss.

In the low field Stage 1 VLHC, we will consider a distributed NEG strip plus lumped ion or cryo pump system in a pumping antechamber connected to the beam tube with long slots.

The non-evaporable getter concept, similar to LEP, is a relatively simple solution, a single NEG strip running inside the pumping antechamber, but needs activation, reconditioning and significant lumped pumping for methane. Since the critical energy for the Stage 1 VLHC is so 
Table 3. Numerical bounds on beam tube pressure Stage 1, ambient room temperature equivalent pressure

\begin{tabular}{ccc}
\hline gas & $\overline{\mathrm{P}}_{\mathrm{j}}$ (nTorr) $\left[\tau_{g}=5 \tau_{L}\right]$ & $\mathrm{P}_{\mathrm{j}}$ (nTorr) $[0.1 \mathrm{~W} / \mathrm{m}]$ \\
\hline \hline $\mathrm{H}_{2}$ & 4 & 76.6 \\
$\mathrm{CH}_{4}$ & 0.54 & 12.6 \\
$\mathrm{H}_{2} \mathrm{O}$ & 0.45 & 12.3 \\
$\mathrm{CO}$ & 0.33 & 8 \\
$\mathrm{CO}_{2}$ & 0.2 & 5 \\
\hline
\end{tabular}

low, $86 \mathrm{eV}$, high $\mathrm{Z}$ shielding (e.g. a layer of $\mathrm{Pb}$ ) around the beam tube vacuum chamber is not needed.

We assume lumped ion pumps, with pumping speed $S=100 \mathrm{l} / \mathrm{s}$, are connected to the pumping antechamber at an axial interval of $\mathrm{L}=22.5$ meters. The effective cylindrical diameter of the antechamber is $8.3 \mathrm{~cm}$. The pumping speed of the lumped ion pumps will be conductance limited by the beam tube and the antechamber, and the effective pumping speed for $\mathrm{CH}_{4}$ is then $S_{\text {eff }} 2.2$ $l / s-m$. In our calculations a NEG St101 strip, supplemented by ion pumps for pumping methane, has been considered. The lumped ion pumps will contribute significantly to the overall cost of the vacuum system so it is desirable to look for ways to eliminate them. It has been suggested that NEG strips exposed to photo and secondary electrons may effectively pump methane [6]. Research and development is needed to evaluate this suggestion.

The dependence of the photodesorption yield, number of molecules desorbed per incident photon, for each species “ $\mathrm{j}$ ”, with the normalized photon dose D in units of $10^{20} \mathrm{ph} / \mathrm{m}$, is given by:

$$
\eta_{j}=\eta_{0 j} \mathrm{D}^{-v_{\mathrm{j}}}
$$

where for practical use of the formula we have assumed a constant value of the photodesorption yield $\eta_{0 j}$, up to an integrated photon dose of $\sim 10^{19} \mathrm{ph} / \mathrm{m}$ [7]. The photodesorption coefficients are key parameters for the beam tube vacuum in a storage ring. For the calculations in this paper the data given in Ref [2], obtained for in situ baked $\mathrm{Al}$ at a critical photon energy of $86 \mathrm{eV}$, have been fit with Eqn. 1. The inverse power dependence of the photodesorption yield on the photon dose implies the so called conditioning effect, or the decreasing photodesorption yield due to the removal of gas molecules from the near surface oxide layer with continued exposure to photons. As a result, the required pumping speed decreases in time as a function of the integrated current intensity. This can have implications for the frequency of NEG regeneration.

We will estimate the $\mathrm{CO}$ equivalent pumping speed necessary to achieve the vacuum pressure indicated in Table 3, within a reasonably short conditioning time. We may define as "reasonably short" equivalent to a few tenths of a year of operation, e.g. 0.3, when the machine is running at design beam intensity. An operational year is typically taken to be $\sim 10^{7} \mathrm{sec}$. Therefore, we estimate the $\mathrm{CO}$ equivalent pumping speed needed to reach $\tau_{\mathrm{g}}=5 \tau_{\mathrm{L}}$ by $\mathrm{I}_{\mathrm{t}}=158 \mathrm{~A}$-hrs. In Fig. 1 the $\mathrm{CO}$ equivalent pumping speed $\mathrm{S}_{\mathrm{CO}}$ required to achieve a beam-gas scattering lifetime $\tau_{\mathrm{g}}$ is plotted as a function of the integrated current for $\tau_{\mathrm{g}} / \tau_{\mathrm{L}}=0.01,0.03,0.1,0.3,1,3$ and 10. From Fig. 1 we may conclude that a pumping speed $S=32 \mathrm{l} / \mathrm{s}-\mathrm{m}$ is required to reach a beam-gas scattering lifetime of $\tau_{\mathrm{g}}=5 \tau_{\mathrm{L}}$ after an integrated beam current $\mathrm{I}{ }^{*} \mathrm{t}=158 \mathrm{~A}$-hrs for the Stage 1 VLHC. For more detailed calculation and formulae, see Ref. 1 and the future reports given in the abstract of this paper. 


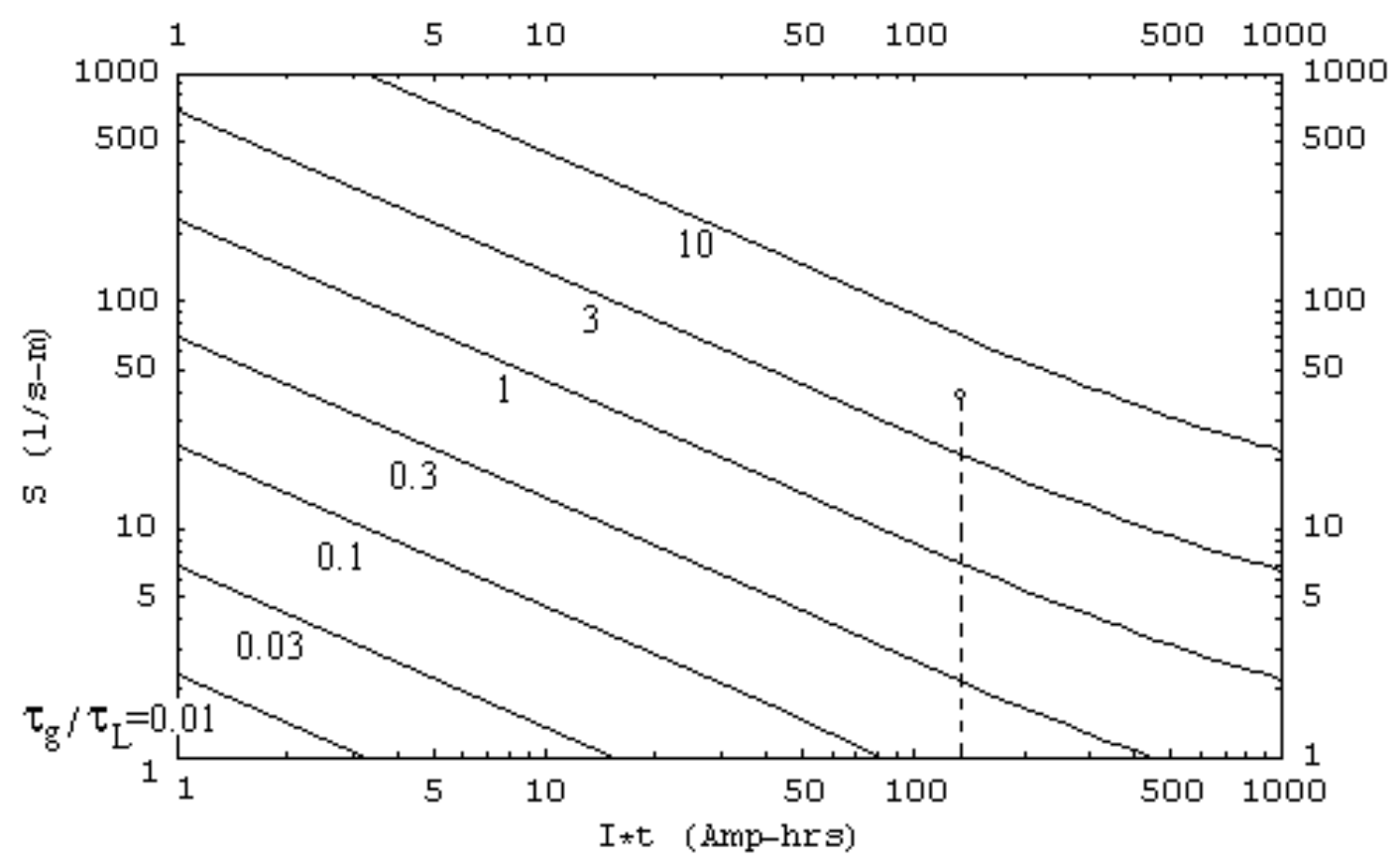

Fig.1. Pumping speed required, in Stage 1, to achieve a specified beam-gas scattering luminosity lifetime versus integrated current I $*$ t, from $\tau_{\mathrm{g}} / \tau_{\mathrm{L}}=0.01$ to 10 , with $\tau_{\mathrm{L}}=46.5 \mathrm{hrs}$. Assuming a beamgas scattering lifetime much larger than the luminosity lifetime, $\tau_{\mathrm{g}} / \tau_{\mathrm{L}}=5$, and $\mathrm{I} * \mathrm{t}=158 \mathrm{~A}$-hrs $(1 / 3$ of a year of operation), the required pumping speed is $S \sim 32 \mathrm{l} / \mathrm{s}-\mathrm{m}$, for $\mathrm{H}_{2}, \mathrm{CO}$ and $\mathrm{CO}_{2}$, while the required pumping speed for $\mathrm{CH}_{4}$ is $S \sim 1.55 \mathrm{l} / \mathrm{s}-\mathrm{m}$.

\section{Conditioning Scenario}

The conditioning effect will improve the beam lifetime which is itself then function of the integrated current intensity. We present here a possible conditioning scenario, for Stage 1 VLHC, where we increase the initially injected beam current from $50 \mathrm{~mA}$ up to $190 \mathrm{~mA}$ (nominal), in four steps. Our procedure is to inject the beam, firstly with increased current, then with current at nominal value, whenever the luminosity lifetime reaches $\tau_{\mathrm{L}}=46.5 \mathrm{hrs}$, corresponding to the design parameter value. The possible beam current program for our conditioning scenario is plotted in Fig. 2.

The beam lifetime $\tau_{\mathrm{b}}$ is related to the vacuum pressure and to the pp collision rate at the interaction point, and is defined as $1 / \tau_{\mathrm{b}}=1 / \tau_{\mathrm{pp}}+1 / \tau_{\mathrm{g}}$. In Fig. 3 the beam lifetime is shown as a function of time, for our conditioning scenario. The luminosity lifetime is then given by $\tau_{L}=\tau_{b} / 2$.

The total beam tube vacuum gas pressure decreases as the surface is progressively cleaned, and is shown in Fig. 4 as the $\mathrm{CO}$ scattering equivalent pressure. The equivalent $\mathrm{CO}$ scattering pressure of gas species " $\mathrm{j}$ " is defined as:

$$
P_{s, j}=P_{j} \frac{\sigma_{j}}{\sigma_{C O}}
$$

and is also shown for each gas in Fig. 4. The CO scattering equivalent gas pressure of species "j" is equal to the partial $\mathrm{CO}$ gas pressure which would result in the same beam-gas scattering as the actual pressure of species " $j "$. In this way the partial pressures in Fig. 4 can be compared on an equal footing as they effect the beam-gas scattering lifetime. We can see from Fig. 4 that $\mathrm{CH}_{4}$ is the gas having the largest equivalent $\mathrm{CO}$ scattering pressure. The pumping speed of the NEG material decreases in time as the pumped gas molecules accumulate on the surface. For this reason we have taken into account, in our calculations, the pumping speed variation of the NEG 


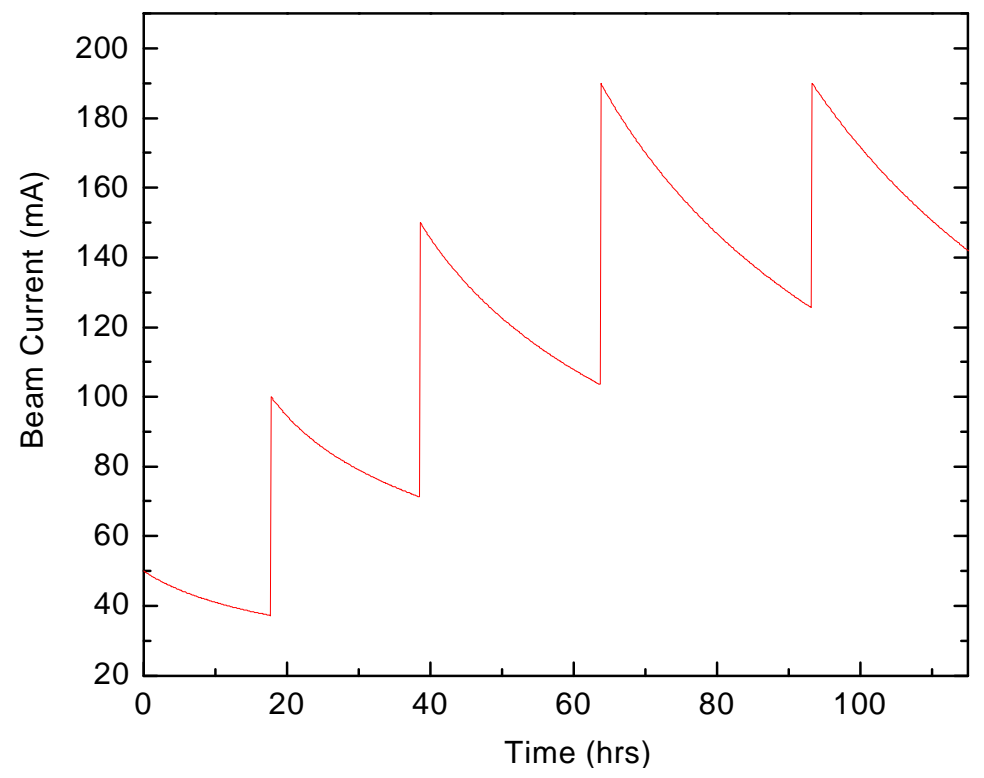

Fig.2. Beam current intensity possible scenario, during the conditioning period in the VLHC, Stage 1, increasing the current from $50 \mathrm{~mA}$ to $190 \mathrm{~mA}$ (nominal), in four steps.

St101 strip (30 $\mathrm{mm}$ wide, LEP type) as a function of the quantity of gas that has been pumped as given in Ref. [4]. Once the NEG surface saturates, a surface regeneration will be necessary to reestablish the maximum initial pumping speed. The surface pumping capacity for $\mathrm{CO}$ is $10^{-4}$ Torr $-1 / \mathrm{cm}^{2}$ [3]. We therefore have to consider reconditioning the NEG after a total desorbed gas load of Q 3 $10^{-2}$ Torr-l/m [4]. As an example, after $200 \mathrm{hrs}$ at the end of this initial conditioning scenario, the total $\mathrm{CO}$ and $\mathrm{CO}_{2}$ gas amount pumped by the NEG is Q 2.5 $10^{-2}$ Torr$\mathrm{l} / \mathrm{m}$, just below the gas load needed for reconditioning.

\section{Beam scattered power at the interaction point}

In addition to previous considerations on the beam tube vacuum issues for the VLHC, we have estimated the beam scattered power at the IP, as a result of the pp collisions, which is given by

$$
P_{I P}=\sigma_{p p} \cdot L \cdot E
$$

where $\mathrm{L}$ is the luminosity. The beam scattered power per beam is shown in Table 4, and compared to the LHC case.

\section{References}

[1] W. Turner "Beam Tube Vacuum in Low Field and High Field Very Large Hadron Collider", LBNL39482, UC414, presented at the Snowmass Conference, CO, October 1996.

[2] J. Gomez-Goni, O. Gröbner and A.G. Mathewson "Exposure of a baked Al lined stainless steel chamber to photons", CERN, Vacuum Technical Note 94-10 pg. 9, AT-VA/JGG.

[3] A. Chao and Tigner, "Handbook of Accelerator Physics and Engineering", pg.329

[4] C. Benvenuti, "Molecular Surface pumping: the getter pumps", CAS CERN Accelerator School Vacuum Technology, CERN Yellow book, pg. 48.

[5] W. Foster and al. "Staged VLHC Parameters List, version 2/23/01", private communication, Fermilab, Chicago, IL, February 2001.

[6] K. Kennedy, private communication, LBNL, Apr. 2001.

[7] O. Gröbner, "Dynamic outgassing", CAS CERN Accelerator School - Vacuum Technology, CERN Yellow book, pg. 130. 


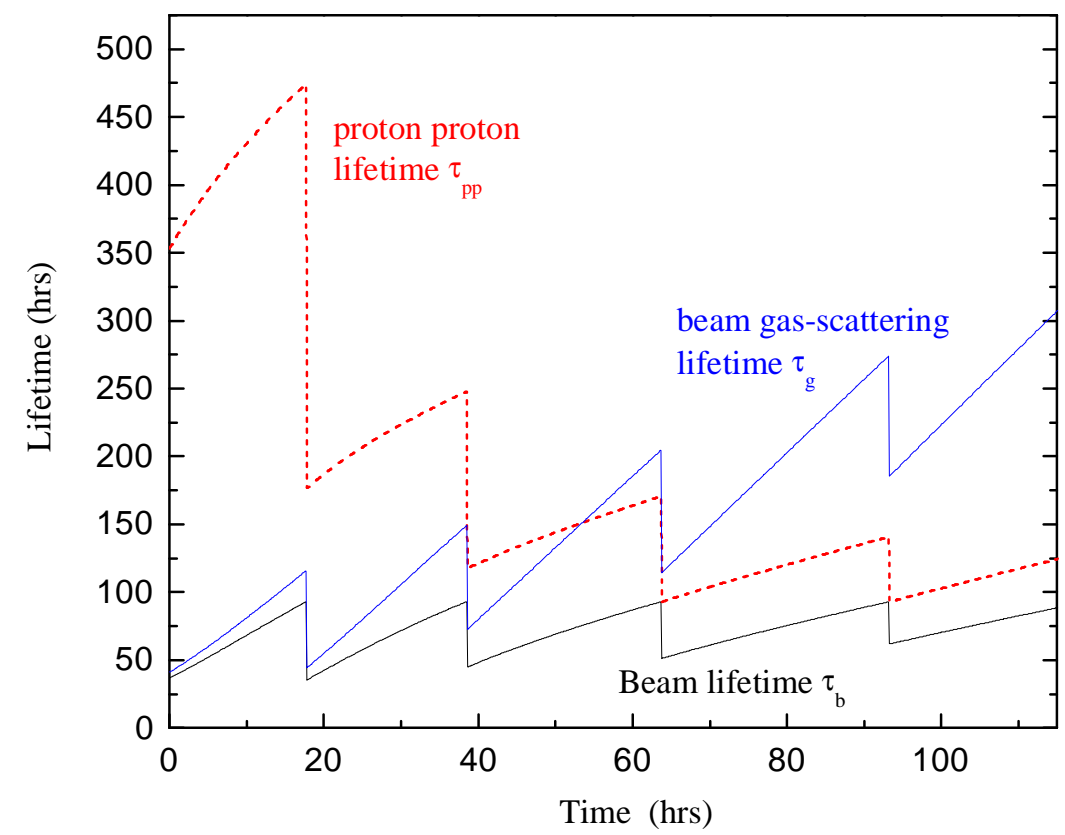

Fig.3. Beam lifetime, beam-gas scattering lifetime, proton-proton lifetime in this possible conditioning scenario.

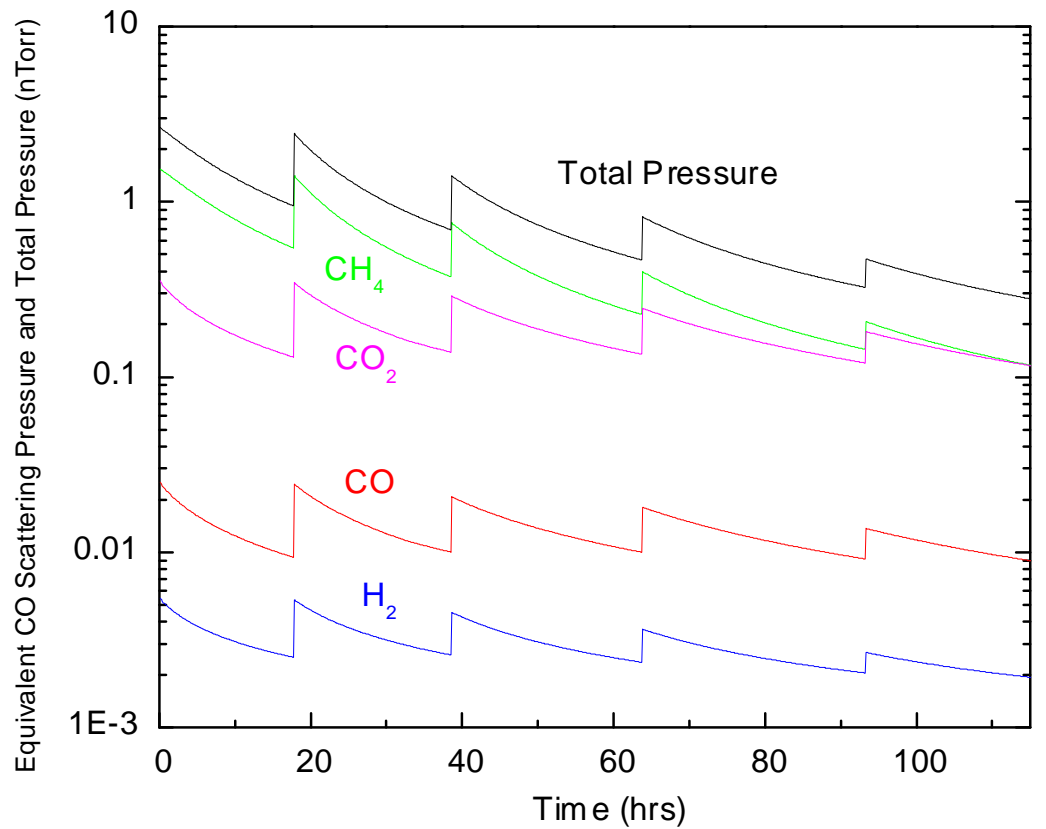

Fig. 4. Equivalent to $\mathrm{CO}$ scattering pressure and total pressure in this possible conditioning scenario.

Table 4. Beam scattered power per beam at the interaction point for Stage 1 and Stage 2 compared to the $\mathrm{LHC}$, where $\sigma_{\mathrm{pp}}$ is the total, elastic plus inelastic, pp cross section.

\begin{tabular}{llccc}
\hline & $\sigma_{\mathrm{pp}}(\mathrm{mb})$ & $\mathrm{L}\left(\mathrm{cm}^{-2} \mathrm{~s}^{-1}\right)$ & $\mathrm{E}_{\text {beam }}(\mathrm{TeV})$ & $\mathrm{P}_{\mathrm{IP}}(\mathrm{kW})$ \\
\hline \hline LHC & 120 & $10^{34}$ & 7 & 1.3 \\
VLHC Stage 1 & 136 & $10^{34}$ & 20 & 4.3 \\
VLHC Stage 2 & 178 & $2 \times 10^{34}$ & 87.5 & 50 \\
\hline
\end{tabular}

